\title{
IMAGE RECONSTRUCTION FROM PHASED-ARRAY MRI DATA BASED ON MULTICHANNEL BLIND DECONVOLUTION
}

\author{
Huajun She ${ }^{1}$, Rong-Rong Chen ${ }^{1}$, Dong Liang ${ }^{2}$, Yuchou Chang ${ }^{2}$, Leslie Ying ${ }^{2}$ \\ ${ }^{1}$ Department of Electrical and Computer Engineering, University of Utah, Salt Lake City, UT, USA \\ ${ }^{2}$ Department of Electrical Engineering and Computer Science, University of Wisconsin-Milwaukee, Milwaukee, WI, USA
}

\begin{abstract}
In this paper we consider image reconstruction from multichannel phased array MRI data without prior knowledge of the coil sensitivity functions. A new framework based on multichannel blind deconvolution (MBD) is developed for joint estimation of the image function and the sensitivity functions in $k$-space. By exploiting the smoothness of the estimated functions in the spatial domain, we develop a regularization approach in conjunction with MBD to obtain good reconstruction of the image function. Experimental results using simulated and real data demonstrate that the proposed reconstruction algorithm can better removes the sensitivity weighting in the reconstructed images compared to the sum-of-squares (SoS) approach.
\end{abstract}

Index Terms - Phased array MRI, multichannel deconvolution, regularization, image restoration

\section{INTRODUCTION}

MRI using phased array coils [1] have emerged as a technique to improve the signal-to-noise ratio (SNR) of the image or reduce the image acquisition time [2-4]. Although optimal SNR is achieved with knowledge of the coil sensitivity functions, these sensitivity functions are usually unknown and need to be estimated from some calibration data [5]. A typical method to reconstruct the original image without knowledge of the sensitivity functions is the sumof-squares (SoS) method [3,4]. In SoS, the image is reconstructed from multiple images acquired with phased array coils by taking the square root of the sum of the absolute squares of the images acquired at each channel. The SoS method is based on the assumption that the sum of the absolute squares of all sensitivity functions are spatially uniform such that the spatially varying sensitivity weighting is removed. However, this assumption is usually violated with surface coils, which causes the reconstructed image to be dark at locations further away from all coils. The nonuniformity of the image intensity greatly complicates further automatic analysis such as registration and tissue segmentation [6]. Some works [5,7] have studied joint estimation of both the image and sensitivity functions to improve the image quality. For example, Ref. [7] models the sensitivity functions as a polynomial in the spatial domain and estimates sensitivity functions based on a multichannel blind deconvolution (MBD) framework [7-9]. Although the primary objective of Ref. [7] is to better estimate the sensitivity functions for SENSE reconstruction [3], the image is reconstructed at the same time. A limitation with the method is that when the object mask is not accurate enough to remove the noise in background, the reconstructed image may still suffer from non-uniformity.

In this paper, we propose a new approach to jointly estimate the image and sensitivity functions in $k$-space. The approach is also based on a MBD framework, but is different from Ref. [7] in that the convolution in the proposed method is in $k$-space and is a circular one. The proposed method only needs the prior information that the image and sensitivity functions are smooth in the spatial domain, but avoids the need for an accurate object mask.

\section{PROBLEM FORMULATION}

In MRI with phased array coils, the $k$-space data are acquired simultaneously from $P$ different receiver coils. The acquisition equation is

$$
Y_{i}\left(k_{x}, k_{y}\right)=\iint f(x, y) h_{i}(x, y) e^{-\mathrm{j} 2 \pi\left(k_{x} x+k_{y} y\right)} d x d y
$$

where $(x, y)$ are spatial domain coordinates, $\left(k_{x}, k_{y}\right)$ are $k$ space domain coordinates, $f(x, y)$ is the desired object image, $Y_{i}\left(k_{x}, k_{y}\right)$ are the $k$-space sampling data for the $i$-th coil, $h_{i}(x, y)$ are the sensitivity functions for the $i$-th coil $(i=1,2, \cdots P)$.

Eq. (1) is the Fourier transform of the sensitivityweighted images $y_{i}(x, y)=f(x, y) h_{i}(x, y)$, or its discrete representation

$$
y_{i}(m, n)=f(m, n) h_{i}(m, n) .
$$

From the property of discrete Fourier transform (DFT) we know that multiplication in spatial domain corresponding to the circular convolution in $k$-space domain. So we have

$$
Y_{i}\left(k_{m}, k_{n}\right)=F\left(k_{m}, k_{n}\right) * H_{i}\left(k_{m}, k_{n}\right),
$$

where " $*$ " is 2-D circular convolution. This formulation is similar to the MBD problem shown in Figure 1. 


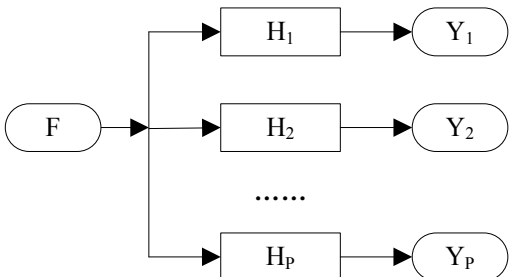

Fig. 1. The multichannel blind deconvolution model.

In order to recover both the $k$-space image $F\left(k_{m}, k_{n}\right)$ and sensitivity functions $H_{i}\left(k_{m}, k_{n}\right)$ from $Y_{i}\left(k_{m}, k_{n}\right)$, the image reconstruction problem can be handled in the MBD framework, which considers the outputs to be known, but the input and filters all unknowns to be reconstructed.

\section{PROPOSED METHOD}

In this section, we describe the proposed method for computing $F\left(k_{m}, k_{n}\right)$ and $H_{i}\left(k_{m}, k_{n}\right)$ which combines the subspace method for MBD [9] and the regularization approach [10]. In Section 3.1, we review the subspace approach and present it for the case of circular convolution. In Section 3.2 we show that solution to the subspace approach is not unique, which leads to the regularization approach discussed in Section 3.3. As shown in Section 3.4, we follow an alternative minimization technique to iteratively compute the $F\left(k_{m}, k_{n}\right)$ and $H_{i}\left(k_{m}, k_{n}\right)$. Issues related to efficient computations of some quantities required for optimization procedure are discussed in Section 3.5.

\subsection{Subspace Approach}

The subspace approach for the MBD problem comes from the cross relationship [9], which is valid for any two pairs of filters and outputs shown in Figure 1:

$$
Y_{i} * H_{j}-Y_{j} * H_{i}=0 \text {. }
$$

Let $\overrightarrow{H_{i}}=\operatorname{vec}\left\{H_{i}\right\}$ denote a column vector consisting of stacked columns of $H_{i}$. Then the stacked column vectors of $\left\{Y_{i} * H_{j}\right\}$, denoted by $\operatorname{vec}\left\{Y_{i} * H_{j}\right\}$, can be written as

$$
\operatorname{vec}\left\{Y_{i} * H_{j}\right\}=\mathfrak{C}_{\left(M_{j}, N_{j}\right)}\left\{Y_{i}\right\} \overrightarrow{H_{j}} .
$$

Here $\mathfrak{C}_{\left(M_{j}, N_{j}\right)}\left\{Y_{i}\right\}$ is the 2-D circular convolution matrix that is dependent on the dimensions $\left(M_{j}, N_{j}\right)$ of the filter $H_{j}$. Hence, we can rewrite (4) in a product form as

$$
\mathfrak{C}_{\left(M_{j}, N_{j}\right)}\left\{Y_{i}\right\} \overrightarrow{H_{j}}-\mathfrak{C}_{\left(M_{i}, N_{i}\right)}\left\{Y_{j}\right\} \overrightarrow{H_{i}}=0 .
$$

In the following, for brevity we suppress $\left(M_{j}, N_{j}\right)$ in $\mathfrak{C}_{\left(M_{j}, N_{j}\right)}\left\{Y_{i}\right\}$ and simply denote it by $\mathfrak{C}^{\mathfrak{c}}\left\{Y_{i}\right\}$. Next, we let $\vec{H}=\left[\begin{array}{lll}{\overrightarrow{H_{1}}}^{T} & \ldots & {\overrightarrow{H_{P}}}^{T}\end{array}\right]^{T}$, where $(\cdot)^{T}$ represents the conjugate transpose operator. Since there are a total of
$P(P-1) / 2$ cross relationship, we put them in a matrix form as

$$
\begin{aligned}
& \mathcal{Y} \vec{H}=0, \\
& \text { where } \mathcal{Y}=\left(\begin{array}{lll}
\mathcal{Y}_{1}^{T} & \ldots & \mathcal{Y}_{P-1}^{T}
\end{array}\right)^{T} \text { and }
\end{aligned}
$$

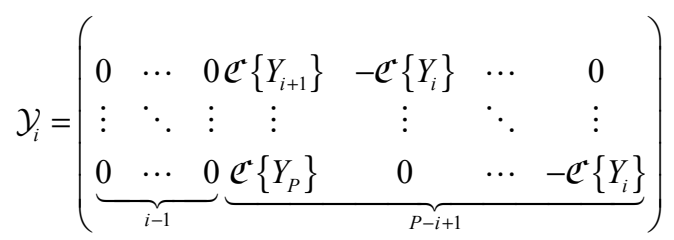

Equation (7) shows that the filter coefficients $\vec{H}$ must belong to the null subspace of $\mathcal{Y}$.

\subsection{Non-uniqueness of Subspace Method}

In this section, we use a simple example to show that solution of the subspace method (7) is not unique. We consider the SoS image as

$$
f_{\text {sos }}(m, n)=\sqrt{\sum_{i=1}^{P}\left|y_{i}(m, n)\right|^{2}},
$$

and the corresponding sensitivity function is given by

$$
h_{\text {sos }, i}(m, n)=\frac{y_{i}(m, n)}{f_{\text {sos }}(m, n)}, i=1, \cdots, P \text {. }
$$

It follows that

$$
y_{i}(m, n)=f_{\text {sos }}(m, n) h_{s o s, i}(m, n) .
$$

We then take the DFT to obtain

$$
Y_{i}\left(k_{m}, k_{n}\right)=F_{\text {sos }}\left(k_{m}, k_{n}\right) * H_{s o s, i}\left(k_{m}, k_{n}\right)
$$

From (12), one can verify that the following cross relationship still holds:

$Y_{j}\left(k_{m}, k_{n}\right) * H_{s o s, i}\left(k_{m}, k_{n}\right)=Y_{i}\left(k_{m}, k_{n}\right) * H_{s o s, j}\left(k_{m}, k_{n}\right)$.

Hence, we have

$$
\mathcal{Y} \overrightarrow{H_{s o s}}=0 \text {. }
$$

Next, we can multiply a particular pixel of the SoS image $f_{\text {sos }}(m, n)$ by $1 / 2$, and multiply the corresponding pixel of sensitivity function $h_{s o s, i}(m, n)$ by 2 to get a new pair of $f_{\text {sos }}(m, n)$ and $h_{\text {sos }, i}(m, n)$. Since (11) still holds for the new pair, it is also a solution to (14). This confirms that we have an infinitely many solutions to (14). To resolve such ambiguity problem, in the next section we propose a regularization approach to utilize the smoothness of the image function and the sensitivity functions in the spatial domain.

\subsection{Regularization}

We propose to estimate the image $\vec{F}$ and sensitivity functions $\vec{H}$ through minimizing the energy function defined as

$$
\begin{aligned}
E(\vec{F}, \vec{H})= & \sum_{i=1}^{P}\left\|\mathcal{C}^{2}\left\{H_{i}\right\} \vec{F}-\vec{Y}\right\|^{2} \\
& +\beta_{1}\|\mathcal{Y} \vec{H}\|^{2}+\alpha Q(\vec{F})+\beta_{2} R(\vec{H})
\end{aligned}
$$


In the first term we have $\vec{F}=\operatorname{vec}\{F\}$ and $\vec{Y}_{i}=\operatorname{vec}\left\{Y_{i}\right\}$. This term measures the fidelity to the data and comes from our acquisition model in (3). The second term comes from the subspace approach. Note that we add the last two regularization terms to utilize the smoothness of the image and the sensitivity functions in the spatial domain. The positive constants $\beta_{1}, \alpha$ and $\beta_{2}$ are adjusted to account for the penalty of violating each of the latter three terms in (15). The third term $Q(\vec{F})$ is a smoothing term of the object image in spatial domain. We use total variation to do regularization, which in the continuous case corresponds to $Q(\vec{F})=\int|\nabla f|$. Because of the nonlinearity of total variation, we use half-quadratic algorithm in [11] to overcome the nonlinearity of the total variation operation. After the discretization, we have

$$
Q(\vec{F})=(W \vec{F})^{T} \widetilde{L}_{F}(W \vec{F})=\vec{F}^{T} L_{F} \vec{F},
$$

where $\widetilde{L}_{F}$ is a positive semi-definite block tridiagonal matrix whose elements depend on the gradient of the image, $W$ is the inverse Fourier transform matrix to transform the $k$-space image data $F$ to the spatial domain. The forth term $R(\vec{H})$ is a smoothing term of the sensitivity functions in the spatial domain. It is defined as

$$
\begin{aligned}
& R(\vec{H})=\sum_{i=1}^{P}\left(W \vec{H}_{i}\right)^{T} \widetilde{L}_{H_{i}}\left(W \vec{H}_{i}\right) \\
& =(\vec{H})^{T}\left[\begin{array}{ccc}
W^{H} L_{H_{1}} W & & \\
& \ddots & \\
& & W^{H} L_{H_{P}} W
\end{array}\right](\vec{H})=(\vec{H})^{T} L_{H}(\vec{H})
\end{aligned}
$$

\subsection{Alternative Minimization}

It follows from (15) that the energy function can be written as

$$
\begin{gathered}
E(\vec{F}, \vec{H})=\sum_{i=1}^{P}\left\|\mathcal{C}^{2}\left\{H_{i}\right\} \vec{F}-\overrightarrow{Y_{i}}\right\|^{2}+\alpha \vec{F}^{T} L_{F} \vec{F} \\
+\beta_{1}\|\mathcal{Y} \vec{H}\|^{2}+\beta_{2} \vec{H}^{T} L_{H} \vec{H}
\end{gathered} .
$$

The energy function $E$ is a function of $\vec{F}$ and $\vec{H}$, and it is not a convex function. However, $E$ is convex with respect to $\vec{F}$ if $\vec{H}$ is fixed, and $E$ is convex with respect to $\vec{H}$ if $\vec{F}$ is fixed. Thus, the optimization problem can be solved by an alternative minimization method [10], which computes a minimization sequence $\left(\vec{F}^{m}, \vec{H}^{m}\right)$ alternatively. In the following two-step approach one computes the derivatives with respect to $\vec{F}^{T}$ and $\vec{H}^{T}$ respectively [12], and set them to be zero to find the minimum point. Step 1)

$$
\begin{aligned}
& \vec{F}^{m}=\arg \min _{\vec{F}} E\left(\vec{F}, \vec{H}^{m-1}\right) \Rightarrow \frac{\partial E}{\partial \vec{F}^{T}}=0 \Leftrightarrow \\
& \left(\sum_{i=1}^{P} \mathfrak{C}^{\mathfrak{c}}\left\{H_{i}\right\}^{T} \mathfrak{C}^{\mathfrak{c}}\left\{H_{i}\right\}+\alpha L_{F}\right) \vec{F}=\sum_{i=1}^{P} \mathfrak{C}\left\{H_{i}\right\}^{T} \vec{Y}_{i}
\end{aligned}
$$

Step 2)

$$
\begin{aligned}
& \vec{H}^{m}=\arg \min _{\bar{H}} E\left(\vec{F}^{m}, \vec{H}\right) \Rightarrow \frac{\partial E}{\partial \vec{H}^{T}}=0 \Leftrightarrow \\
&\left(\left[I \otimes \mathfrak{C}^{c}\{F\}^{T} \mathfrak{C}\{F\}\right]+\beta_{1} \mathcal{Y}^{T} \mathcal{Y}+\beta_{2} L_{H}\right) \vec{H} \\
& \quad=\left[I \otimes \mathfrak{C}^{c}\{F\}^{T}\right] \vec{Y}
\end{aligned}
$$

where $\otimes$ is kronecker product, $I$ is identity matrix, and $\vec{Y}=\left[\begin{array}{lll}\vec{Y}_{1}^{T} & \ldots & {\overrightarrow{Y_{P}}}^{T}\end{array}\right]^{T}$.

\subsection{Efficient Computation}

In the optimization step (20), the direct computation needs to know $\mathcal{Y}^{T} \mathcal{Y}$ exactly. For large images, $\mathcal{Y}^{T} \mathcal{Y}$ cannot be directly computed because of the large memory requirement. We follow the approach of [9] to compute $\mathcal{Y}^{T} \mathcal{Y}$ as

$$
\begin{aligned}
& U=\mathcal{Y}^{T} \mathcal{Y}=\left\{U_{i, j}\right\}, i, j=1, \ldots, P
\end{aligned}
$$

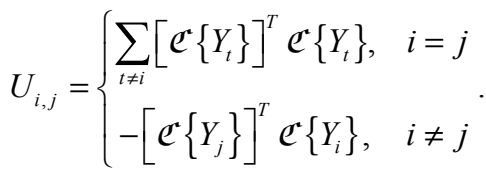

However, it is still prohibitive to store $U_{i, j}$ directly. One way to avoid the matrix computation and storage is to compute $\overrightarrow{G_{i j}}=\mathfrak{C}^{\mathfrak{c}}\left\{Y_{i}\right\} \overrightarrow{H_{j}}$ first, and then use the property of circular convolution matrix

$$
\left[\mathfrak{C}^{c}\left\{Y_{i}\right\}\right]^{T} \overrightarrow{G_{i j}}=\operatorname{vec}\left\{\ddot{Y}_{i} * \operatorname{unvec}\left\{\overrightarrow{G_{i j}}\right\}\right\} .
$$

Here unvec $\{\cdot\}$ converts a vector back to a matrix of appropriate dimensions. Assume $Y_{i}$ has a dimension of $(M, N) \cdot \ddot{Y}_{i}(m, n)$ is the flipped version of $Y_{i}(m, n)$ defined as

$$
\ddot{Y}_{i}(m, n)=Y_{i}(M-m+1, N-n+1) .
$$

This way we avoid direct storage of $U$, and the computation efficiency is increased.

\section{EXPERIMENTAL RESULTS}

Both simulation and in vivo experiments were carried out to evaluate the proposed method. In simulation, a $128 \times 128$ MR image ${ }^{1}$ is used as the original image shown in Figure 2 (a). The simulated $k$-space data were generated by Fourier transforming the images weighted by a set of eight sensitivity functions. The sensitivity functions were simulated using the Biot-Savart law [13]. The proposed method is used to reconstruct the original unweighted image from the multichannel $k$-space data. The parameters used in the simulation are $\beta_{1}=1, \alpha=10^{3}$, and $\beta_{2}=10^{3}$, with a 
random initial guess for both the image and sensitivity functions. The large values of $\alpha$ and $\beta_{2}$ are due to the fact that the image function and sensitivity functions are spatially smooth. Figure 2 (b) and (c) show the reconstructed images using the SoS and the proposed method, respectively. It is seen that the center of the SoS reconstruction is darker and thus has a lower contrast than the proposed method.

To quantitatively evaluate the performance, we define the reconstruction error of $\hat{f}$ as

$$
e(\hat{f})=\frac{\left\|\hat{f}-f_{\text {true }}\right\|^{2}}{\left\|f_{\text {true }}\right\|^{2}} \times 100 / 100
$$

The reconstruction error of the proposed method is $10 \%$, which is improved over the reconstruction error of $16 \%$ for the SoS reconstruction.

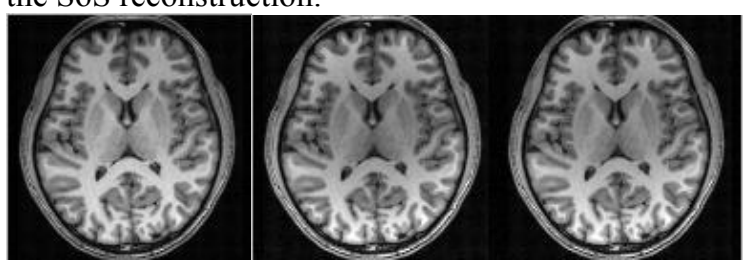

(a)

(b)

(c)

Fig. 2. Simulation results for a 128 by 128 image reconstructed from a set of 8-channel data. (a) True image, (b) SoS reconstruction, (c) reconstruction using the proposed method. Note that the center of (b) is darker than that of (c).

In in vivo experiment, a set of full $k$-space data with the size of $256 \times 256$ were collected using a 4-channel phased array coil. The image is reconstructed using both the SoS and the proposed method. The parameters for the proposed method were $\beta_{1}=1, \alpha=10^{3}$, and $\beta_{2}=10^{10}$, with a random initial guess for both the image and sensitivity functions. $\beta_{2}$ are larger than $\alpha$ due to the fact that the sensitivity functions are much more smooth than the image function in this experiment. Figure 3 (a) and (b) show the reconstructed images using the $\mathrm{SoS}$ and the proposed methods, respectively. It is seen that the intensity of the reconstruction by the proposed method is more uniform across the whole image, when compared with the SoS reconstruction.

\section{CONCLUSION}

We propose a novel regularization-based MBD method to reconstruct the image from multichannel phased-array MRI data. Simulation and experimental results demonstrate that the proposed method can improve the uniformity of the image intensity over the conventional SoS method. The method is expected to be useful in applications where the assumption for SoS reconstruction fails.

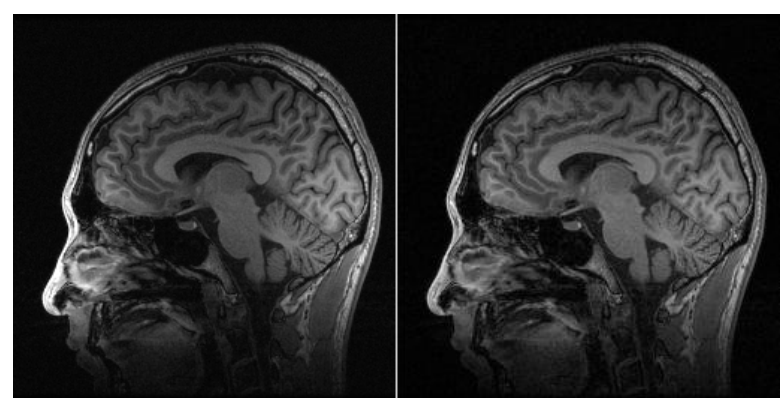

(a)

(b)

Fig. 3. Reconstructions from a set of 4-channel phased array data. (a) SoS reconstruction, (b) reconstruction using the proposed method. Note the intensity of (b) is more uniform than that of (a).

6. ACKNOWLEDGMENT

The authors would like to thank Filip Šroubek for his helpful discussion and for his making available the multichannel blind deconvolution (MBD) MATLAB code at http://zoi.utia.cz/download. This work is supported in part by the National Science Foundation CBET-0731226 and ECS-0547433.

\section{REFERENCES}

[1] P. B. Roemer, W. A. Edelstein, C. E. Hayes, S. P. Souza, and O. M. Mueller, "The NMR phased array," Magn. Reson. Med., vol. 16, pp. 192225, 1990.

[2] D. K. Sodickson and W. J. Manning, "Simultaneous acquisition of spatial harmonics (SMASH): Fast imaging with radiofrequency coil arrays," Magn. Reson. Med., vol. 38, no. 4, pp. 591-603, 1997.

[3] K. P. Pruessmann, M. Weiger, M. B. Scheidegger, and P. Boesiger, "SENSE: Sensitvity encoding for fast MRI," Magn. Reson. Med., vol. 42, pp. 952-962, 1999.

[4] M. A. Griswold, P. M. Jakob, R. M. Heidemann, M. Nittka, V. Jellus, J. Wang, B. Kiefer, and A. Haase, "Generalized autocalibrating partially parallel acquisitions (GRAPPA)," Magn. Reson. Med., vol. 47, pp. 12021210,2002 .

[5] Ying L, and Sheng J., "Joint image reconstruction and sensitivity estimation in SENSE (JSENSE)," Magn. Reson. Med., vol. 57, pp. 11961202, 2007.

[6] Stathis Hadjidemetriou, Colin Studholme, Susanne Mueller, Michael Weiner, and Norbert Schuff, "Restoration of MRI data for intensity nonuniformities using local high order intensity statistics," Medical Image Analysis, vol. 13, pp. 36-48, 2009.

[7] Robert L. Morrison, Mathews Jacob, and Minh N. Do, "Multichannel estimation of coil sensitivities in parallel MRI," IEEE International Symposium on Biomedical Imaging, 2007.

[8] M. Gurelli and C. Nikias, "EVAM: An eigenvector-based algorithm for multichannel blind deconvolution of input colored signals," IEEE Trans. on Signal Processing, vol. 43, no. 1, pp. 134-149, 1995.

[9] G. Harikumar and Y. Bresler, "Blind restoration of images blurred by multiple filters: Theory and efficient algorithms," IEEE Trans. on Image Processing, vol. 8, no. 2, pp. 202-219, 1999.

[10] Filip Šroubek and Gabriel Cristóbal, "A Unified Approach to Superresolution and Multichannel Blind Deconvolution," IEEE Trans. on Image Processing, vol. 16, no. 9, pp. 2322-2332, 2007.

[11] G. Aubert and P. Kornprobst, Mathematical Problems in Image Processing, New York: Springer Verlag, 2002.

[12] Brandwood, D.H., "A complex gradient operator and its application in adaptive array theory," Communications, Radar and Signal Processing, IEE Proceedings $F$, vol. 130, issue 1, pp. 11-16, 1983.

[13] Ulaby F.T., Fundamentals of applied electromagnetics, 5th Edition, Prentice Hall, 2006. 